\title{
Thinking inside the box: have we underestimated cardiac rehabilitation?
}

\author{
Authors: Henna Shakoor ${ }^{A}$ and Safina Begum ${ }^{A}$
}

Cardiac rehabilitation (CR) is a comprehensive secondary intervention programme offered to select cardiac patients in order to educate them on their condition, modify cardiovascular risk factors and improve functional capacity.

There is a large body of evidence demonstrating the extensive benefits of attending a CR programme with significant reduction in cardiac morbidity and mortality, reduction in cardiac symptoms, enhanced psychosocial wellbeing and improved health-related quality of life. Despite this welldocumented data, nationally only $50 \%$ of all eligible patients participate in CR. The reasons for this are multifactorial and include both patient and clinician factors.

While CR specifically targets the barriers to essential lifestyle changes that patients experience post-cardiovascular event, the emphasis remains heavily on medical management and invasive intervention alone.
This poster aims to summarise the proven benefits of $\mathrm{CR}$, identify barriers to referral and attendance and offer suggestions to optimise CR uptake and delivery.

In summary, we urge clinicians to 'think inside the box' when approaching the management of patients with cardiovascular disease, providing greater emphasis on lifestyle changes and effective deliverance of structured CR programmes which form the foundation of good prognostic outcomes for such patients.

\section{Conflicts of interest}

None declared. 